\title{
Finite Element Analysis of Bone Resorption Around Dental Implant
}

\author{
Lihe QIAN $^{* *}$, Mitsugu TODO ${ }^{* *}$, Yasuyuki MATSUSHITA ${ }^{* * *}$ \\ and Kiyoshi KOYANO ${ }^{* * *}$ \\ **Research Institute for Applied Mechanics, Kyushu University, \\ 6-1 Kasuga-koen, Kasuga 816-8580, Japan \\ E-mail: dlhqian@yahoo.com or todo@riam.kyushu-u.ac.jp \\ ***Faculty of Dental Sciences, Kyushu University \\ 3-1-1 Maidashi, Higashi-ku, Fukuoka 812-0041, Japan
}

\begin{abstract}
Previously, a few models have been proposed to predict bone resorption process due to stress shielding in long bones such as proximal femur; however, there are almost no reports on finite element analysis of loss of marginal dental bone that is caused mainly by occlusive overload. In this work, the stress, strain and strain energy density (SED) criteria were separately applied to simulate overload-induced bone resorption in a jawbone/implant system by means of the finite element analysis. A simplified dental bone/implant model was created, with the bone composed of a cortical bone and a cancellous bone and the implant having the detailed screw structure. The results demonstrated that the simulations according to the equal SED criterion reproduce bone resorption patterns that are more realistic to actual clinical situations, when compared to the equal stress or strain criterion. It was shown that bone resorption starts initially in the cortical bone around the implant neck, then extends downwards, and lastly enters the cancellous bone after passing through the interface of the cortical and cancellous bone. A symmetric bone resorption pattern was revealed under the condition of axial loading, whereas an asymmetric resorption prototype was demonstrated under the oblique loading condition. Moreover, in the case of oblique loading, bone resorption is faster and the amount of resorbed bone is larger, which leads to more micromotion of the dental implant than in the case of axial loading.
\end{abstract}

Key words: Bone Resorption, Bone Remodeling, Dental Implant, Numerical Simulation, Finite Element Analysis

\section{Introduction}

Long-term success rates have been reported in several long-time follow-up studies on dental implants used for rehabilitation of partially or completely edentulous patients ${ }^{(1,2)}$. However, clinical studies have also indicated the loss of marginal dental bone or complete loss of osseointegration of implants where osseointegration has previously been attained, thus causing micromotion of the implants and decreasing the reliability of implantation ${ }^{(3-6)}$.

One major reason for the loss of marginal bone has been associated with unfavorable loading conditions ${ }^{(6-8)}$, which act on oral implants and are transmitted to the surrounding bone. According to the theory of bone remodeling ${ }^{(9)}$, bone is adapted to the internal mechanical environment by changing its density (internal remodeling) or morphology (external remodeling) under certain loading conditions to which the bone is exposed. Within a certain range of mechanical strains, bone is normally in a state of equilibrium. With 
slightly increasing the strain that exceeds the strain range for maintaining the equilibrium state, the bone becomes mildly overloaded and formation of more bone occurs. If the strain rises further and goes beyond a threshold, which exceeds the bone's load-bearing capacity, fatigue microdamage becomes predominant and overload-induced bone resorption happens subsequently. Nevertheless, if the bone strain falls and stays below a certain minimum value, bone resorption occurs as well due to disuse of the bone. In clinical orthopedics, adaptive bone remodeling as well as bone resorption is frequently observed. In the cases of femoral stems in Total Hip Arthroplasty, for example, the most popular explanation for bone loss is the occurrence of stress shielding, i.e., disuse of the bone. ${ }^{(10)}$. On the contrary, the loss of marginal bone in implant/jawbone systems has been mainly attributed to occlusive overload, even in the absence of plaque-related gingivitis ${ }^{(6)}$. In reality, there has been a volume of convincing clinical evidence that supports the above statement that excessive mechanical loading gives rise to resorption of dental bone ${ }^{(7,8,11)}$.

Owing to the important role of mechanical stress in bone resorption recognized clinically, the alleviation of stress shielding in prosthetic surgery such as total joint replacement and as well the prevention of stress and strain concentrations in dental bone/implant systems are strongly recommended. Therefore, much research effort has been devoted to analyzing bone's stress and strain distributions ${ }^{(12-15)}$, with an attempt to optimize the prosthetic design and to improve the mechanical environment in bone.

Furthermore, several attempts have been made, by means of analytical or numerical approaches, to evaluate the phenomenon of bone remodeling and to predict the dynamic process of bone resorption in proximal femur ${ }^{(10,16)}$, which is assumed to be essential to understanding the causes for the time-dependent loosening of the prosthesis and thus the reliability of the system. However, there are almost no reports on numerical simulations of bone resorption process in implant/jawbone systems. The purpose of the present study is thus to investigate the process of dental bone resorption and the consequences resulting from the resorption of the bone, by reproducing some typical patterns of bone resorption observed in clinical realities, by means of a three-dimensional finite element analysis. This investigation is also attempted to provide some new insight into how the bone is resorbed by adapting to the mechanical environment and to reveal what happens during the dynamic evolution of bone resorption.

\section{Bone Resorption Stimulus and Threshold}

A number of mathematical models have been proposed in order to describe bone remodeling phenomena and predict the process of bone formation or resorption. Most of the models assumed that bone tissue has a homeostatic equilibrium strain state, and the bone structure adapts itself as a function of the difference between the actual and equilibrium strain states ${ }^{(17-19)}$. In the case of external remodeling, for example, bone adds or removes material on the surfaces of the bone, activated by the strain state at the surfaces as a stimulus, according to the equation following Cowin and Van Buskirk ${ }^{(20)}$ :

$$
\frac{d X}{d t}=C_{i j}\left(\varepsilon_{i j}-\varepsilon_{i j}^{0}\right)
$$

where $\boldsymbol{X}$ is the surface position, $e_{i j}$ the actual strain tensor, $e_{i j}{ }^{0}$ the remodeling equilibrium strain tensor and $C_{i j}$ a matrix of remodeling constants. Alternatively, Huiskes et al. ${ }^{(21)}$ used not the strain tensor but strain energy density (SED) as a stimulus to predict bone adaptation in a similar way to that proposed by Cowin and Van Buskirk ${ }^{(20)}$. A difference between the actual SED and a reference equilibrium SED would be the driving force for bone adaptation, as expressed by:

$$
\frac{d X}{d t}=C_{x}\left(U-U_{n}\right)
$$


where $\boldsymbol{X}$ is the surface position, $U$ the actual SED, $U_{n}$ the reference equilibrium SED and $C_{x}$ the constants. It follows from either of the above external remodeling models that bone formation would occur when the stimulus ( $e_{i j}$ or $U$ ) exceeds an equilibrium value, while bone resorption would take place if the stimulus falls below the equilibrium value. It is noted that these models do not consider resorption of the bone induced by overload and thus, were mainly applied to long bones such as proximal femurs, where stress shielding (or disuse of the bone) is the major cause for loss of the bone. On the contrary, in dental bone/implant systems, it is occlusive overload that mainly causes bone resorption ${ }^{(6-8)}$, which is hence the major concern in the present research as opposed to long bones. Here, we intend to focus our effort on bone resorption caused by overload not by stress shielding, that is, the bone will be resorbed only when the mechanical stimulus exceeds a threshold value.

According to Frost ${ }^{(9)}$, the threshold value for overload-induced bone resorption (i.e. microdamage) in a cortical bone is in the range of 2500 3500 $\mu \varepsilon$ (microstrain). This strain range is equivalent to a stress range of 34 48 MPa or a SED range of $43 \sim 84 \mathrm{~kJ} / \mathrm{m}^{3}$ (if the bone is assumed to be elastic with a modulus of $13700 \mathrm{MPa}$ ). For a cancellous bone, however, the microdamage threshold can be different from a cortical bone, and there are no reference data of either stress, or strain, or SED that are available as microdamage threshold. While Weinans et al. and others ${ }^{(10,21)}$ have attempted to model bone adaptation in proximal femurs, the necessary constants in Eqs. 1 and 2 which they used were arbitrarily given and tested until the final simulation result of the remodeling process was considered as realistic. Likewise, in the present research, three computation tests will be performed first to determine a suitable criterion as well as the microdamage threshold for each bone. For this purpose, the same threshold value of strain, stress, or SED will be assigned separately to the cortical bone and cancellous bone equally to examine the applicability of each criterion. The tested criterion as well as the corresponding threshold, which leads to the simulated final bone resorption patterns resembling mostly those observed in clinical situations, will be considered appropriate and applied in the final analysis.

\section{Approaches}

The geometry of the implant/bone system is shown in Fig. 1, which is composed of a single free-standing implant and a simplified jawbone. The jawbone consists of a cortical bone and a cancellous bone. Each bone takes the shape of a rectangular solid. The dimensions of the bone layers are shown in Fig. 1, with the thickness of the upper side of the cortical one being $2 \mathrm{~mm}$, and those of the other sides being $1 \mathrm{~mm}$. The implant has a diameter of $3.7 \mathrm{~mm}$ and a length of $10 \mathrm{~mm}$. And it contains a detailed screw structure, with the smallest screw pitch, height of thread and end width of thread being around 0.8, 0.21

(a)

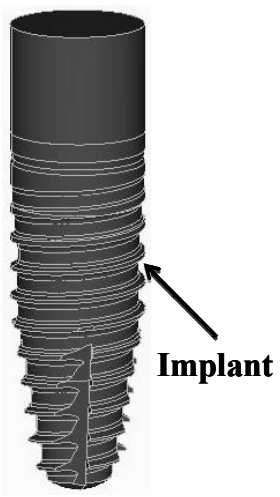

(b)

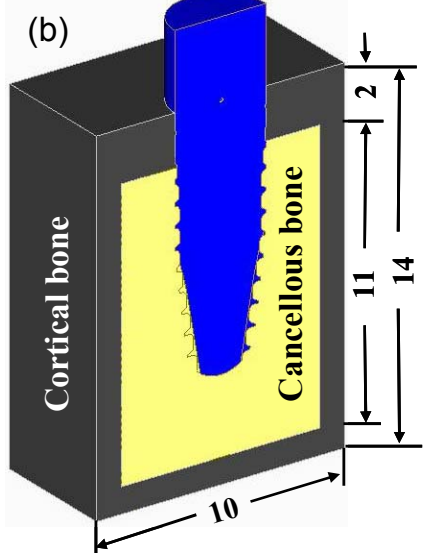

Fig. 1 (a) The geometry of the implant and (b) half of the geometrical model of the implant and two bone layers. Units are in $\mathrm{mm}$. 
and $0.17 \mathrm{~mm}$, respectively.

The finite element mesh of the model is composed of $\sim 80,000$ four-node tetrahedral elements (Fig. 2a). The meshes are gradually coarsened away from the implant/bone interface, with the most refined elements (of the smallest element size of $0.2 \mathrm{~mm}$ ) located near the interface region (Fig. 2b). This smallest element size is of the same order of the size of the screw threads. It should be noted that, before the final model were created, we had performed a convergence test and confirmed that the chosen smallest element size of $0.2 \mathrm{~mm}$ was fine enough to provide sufficiently accurate results for stress/strain analysis. The implant was modeled as an elastic-plastic medium, conforming to von Mises yielding criterion, while the two bones were assigned with isotropic, linear-elastic material properties. The material constants for the implant were taken from pure titanium, i.e., Young's modulus being 106,000 MPa, yield stress $240 \mathrm{MPa}$, and Poisson's ratio 0.30 . The Young's modulus and Poisson's ratio for the cortical bone are 13,700 $\mathrm{MPa}$ and 0.3 , respectively, and for the cancellous bone they are $1370 \mathrm{MPa}$ and 0.3 , respectively ${ }^{(22)}$.

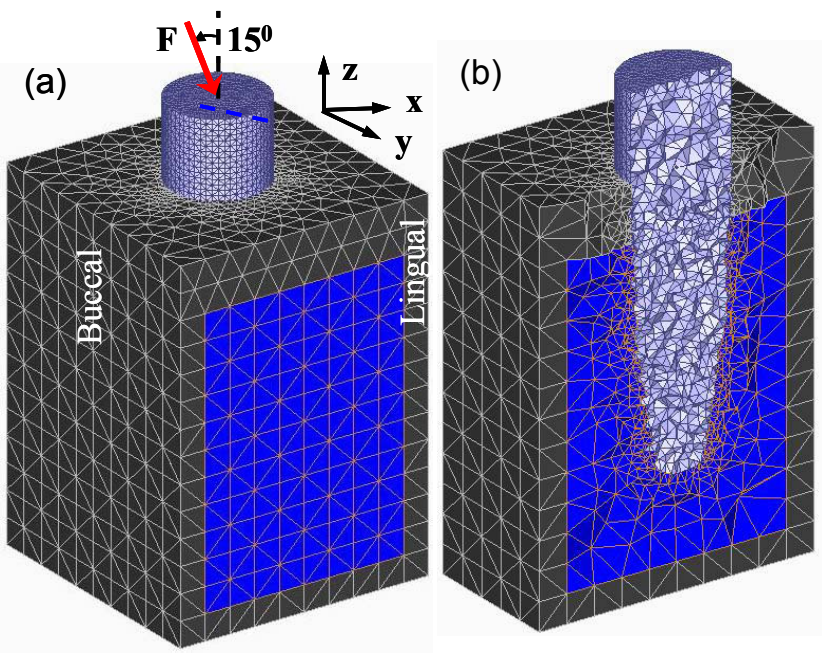

Fig. 2 (a) The entire and (b) half of the finite element meshes of the model.

The interface between the cortical bone and cancellous bone and that between the implant and each bone layer were assumed to be well bonded, corresponding to full osseointegration. The lower surface of the model and the medial and distal planes of the bone were completely constrained. An occlusive load up to $400 \mathrm{~N}$ was gradually applied on the upper surface of the implant within 0.5 seconds. Two loading directions were simulated: one is axial and the other is $15^{\circ}$ bucco-lingually.

As discussed in Section 2, it was necessary to test for a suitable criterion first and to determine the microdamage threshold corresponding to the applied criterion before formal simulation of bone resorption. For testing the equal strain criterion, the same threshold of $2500 \mu \varepsilon$ was assigned to the cortical bone as suggested by Frost ${ }^{(9)}$, and equally to the cancellous bone as well. Similarly, for testing the equal stress and equal SED criteria the same threshold stress of $34 \mathrm{MPa}$ and the same threshold SED of $43 \mathrm{~kJ} / \mathrm{m}^{3}$ were separately assigned to both the cortical bone and cancellous bone equally. And thus, the resorption processes for testing purpose were simulated based on different criteria. In order to realize the simulation of resorption process in the model, the microdamage threshold value was pre-written in the program that was embedded in the LS-DYNA soft package in advance. The stimulus of either stress or strain or SED depending on the selected criterion was calculated locally in each element during computation, and compared with the pre-written threshold. The elements, whose stimulus reached the threshold, were allowed to be removed and thus be resorbed. Such a repeated computation and resorption procedure was continued at various time intervals until the applied loading was stopped. 


\section{Results}

\subsection{Bone resorption processes based on different criteria}

The resorbed bone patterns under the same axial loading simulated based on three different criteria are comparatively shown in Fig. 3. Fig. 3 (a) and (b), respectively, shows the bone patterns at two levels, resorbed according to the strain criterion with the same threshold value of $2500 \mu \varepsilon$ assigned equally to both the cortical and cancellous bone. It can be noted that the bone resorption is initiated in the cancellous bone near the corner of the implant apex. This can be due to the fact that strain concentration occurs initially in these regions. Afterwards, bone resorption proceeds, by following the strain concentration upwards along the interface. Clearly, this type of resorption pattern is not consistent with most actual clinical observations, in which bone resorption tends to start from the upper edge of the cortical bone other than the cancellous bone at the implant tip ${ }^{(7,8)}$.
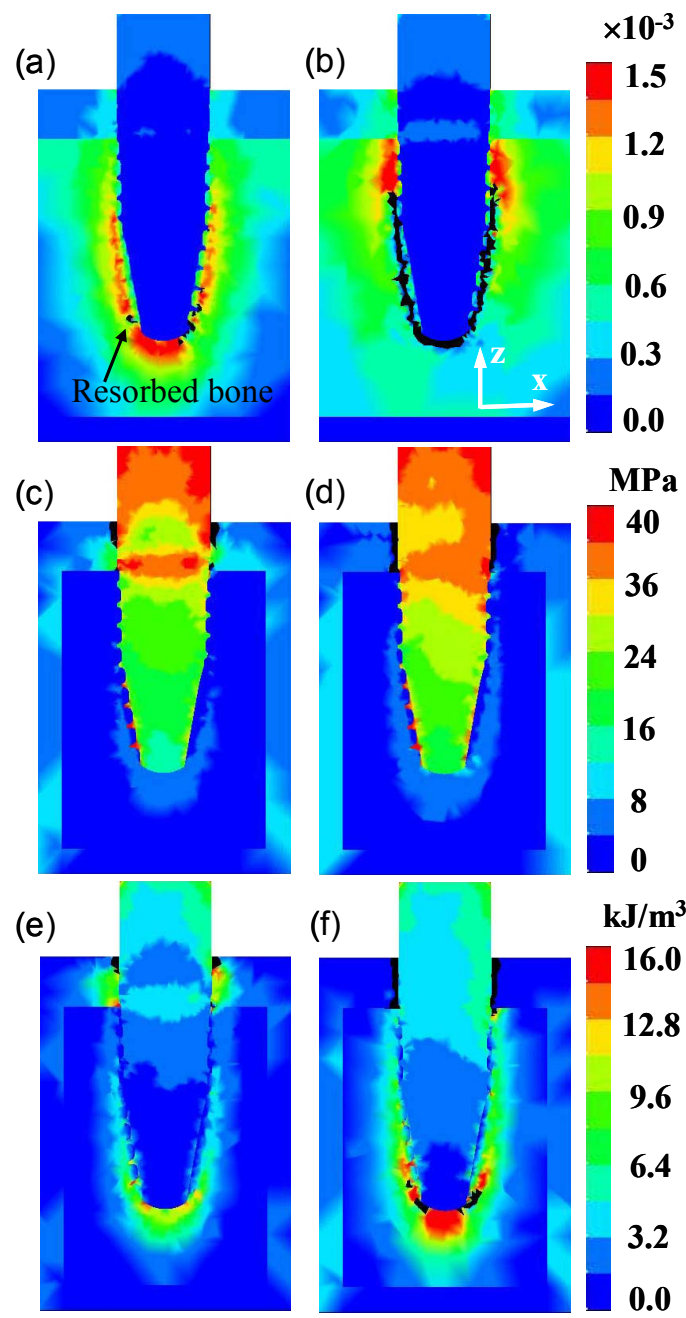

Fig. 3 The bucco-lingual cross sectional patterns of bone at two resorption levels under axial loading simulated according to three different criteria: (a) and (b) the equal strain; (c) and (d) the equal stress;

(e) and (f) the equal SED.

Fig. 3(c) and (d) demonstrates the bone resorption pattern, simulated based on the stress criterion with an identical threshold value of $34 \mathrm{MPa}$ set for the cortical bone and as well the cancellous bone. The bone starts to be resorbed at the upper edge of the cortical bone adjacent to the implant, due to the occurrence of stress concentrations in this region. And 
then, the bone resorption continues towards the cancellous bone. Such features of bone resorption simulated were in agreement with clinical observations ${ }^{(7,8)}$. However, with further increasing loading time, bone resorption almost stops, hardly passing through the interface into the cancellous bone, since the enhanced stress in the cancellous bone could hardly be high enough to reach the pre-set threshold stress value, probably because of its low elastic modulus. This phenomenon may not be as realistic as observed clinically, since resorption of cancellous bone has also been frequently observed in actual implant/jawbone systems ${ }^{(6)}$.

The bone resorption pattern, simulated according to the SED criterion with the same threshold of $43 \mathrm{~kJ} / \mathrm{m}^{3}$ assigned to the cortical bone as well as the cancellous bone, is shown in Fig. 3(e) and (f). Bone resorption occurs initially at the upper edge of the cortical bone in close contact with the implant, and then moves downwards by tracking the concentrated SED. When the cortical bone around the implant is almost fully resorbed, resorption of the bone occurs in the cancellous bone especially at the corners of the implant apex where SED becomes concentrated. This type of bone resorption is in good agreement with in vivo and animal experimental observations ${ }^{(6)}$ and therefore, considered to be realistic. Accordingly, further analysis of bone resorption and the accompanying consequences will be based on simulations according to the equal SED criterion in the following subsections.

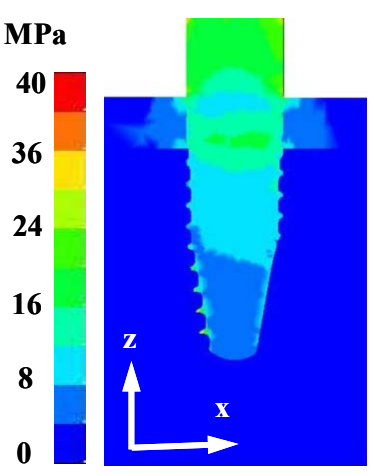

(a) 0.44

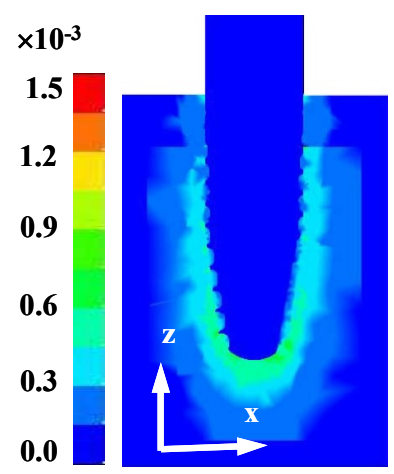

(e) 0.44

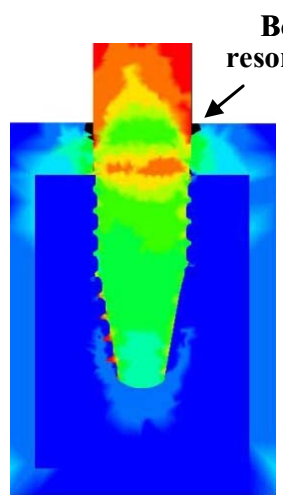

(b) 0.84

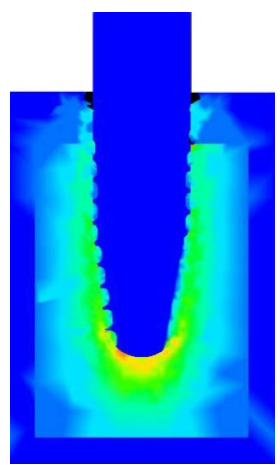

(f) 0.84

Bone

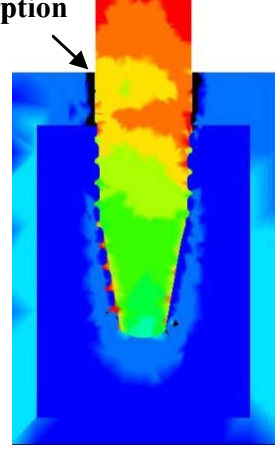

(c) 0.90

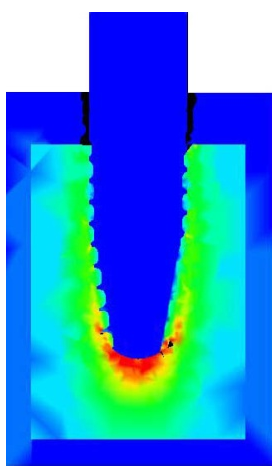

(g) 0.90

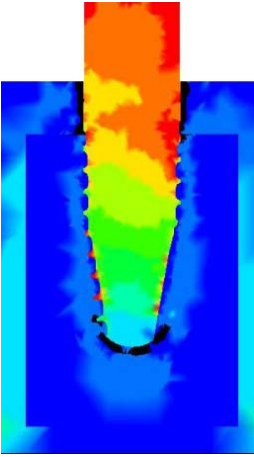

(d) 0.96

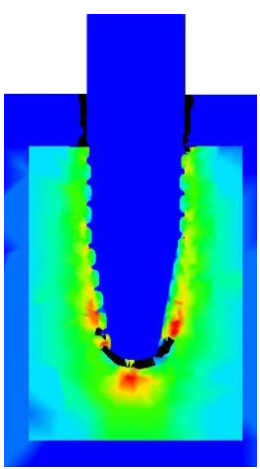

(h) 0.96

Fig. 4 Bucco-lingual cross sectional illustration of the bone resorption patterns under axial loading simulated according to the equal SED criterion, at a normalized simulation time of (a) and (e) 0.44 ; (b) and (f) 0.84 ; (c) and (g) 0.90 ; (d) and (h) 0.96 . The bone patterns are superimposed on the contours of stress (a) (d) and strain (e) (h).

\subsection{Effect of loading angle on bone resorption process}

It can be seen that, in the case of axial loading, dental bone is resorbed almost symmetrically at the circumference of the implant. Figure 4 shows the bucco-lingual 
cross-sectional bone resorption patterns overlapped on the contours of equivalent stress (Fig. 4a d) and strain (Fig. 4e h), at different stages of axial loading. At the beginning, high stress is concentrated at the upper edge of the cortical bone (Fig. 4a) and high strain is concentrated in the cancellous bone near the apex of the implant (Fig. 4e). However, the bone is resorbed initially following the location of stress concentration until the circumferential cortical bone is almost completely resorbed (Fig. 4b, f and c, g). Afterwards, resorption of the bone occurs in the cancellous bone by tracking the location of strain concentration (Fig. 4d and h).

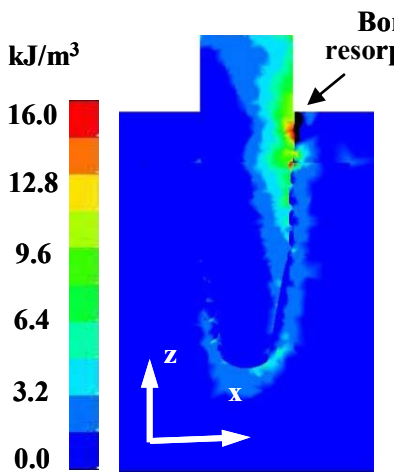

(a) 0.56
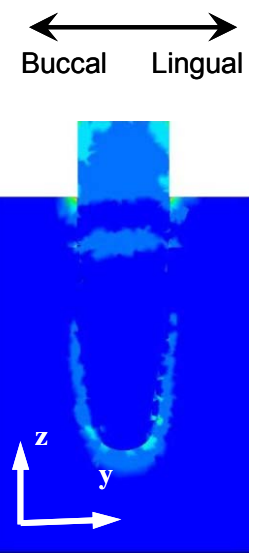

(e) 0.56
Bone

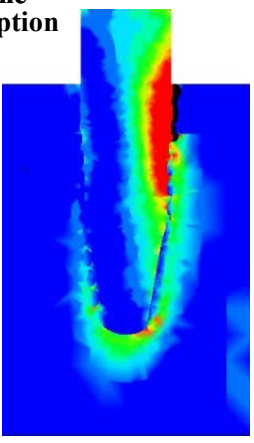

(b) 0.70

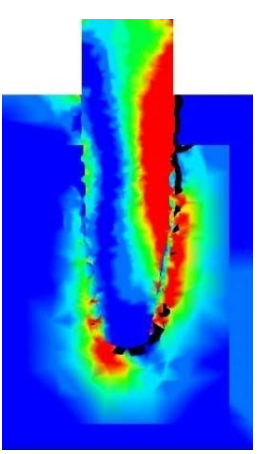

(c) 0.84

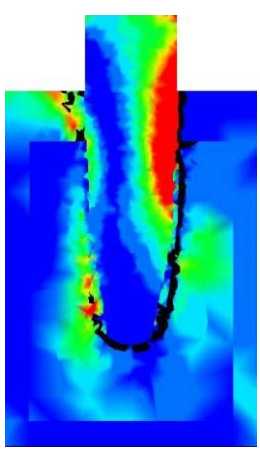

(d) 0.92

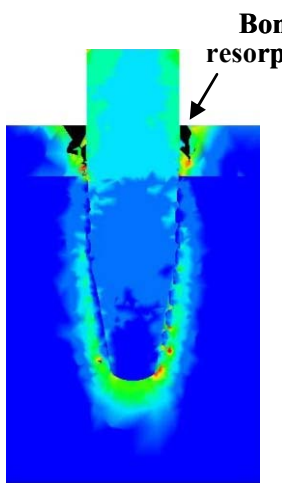

(f) 0.70

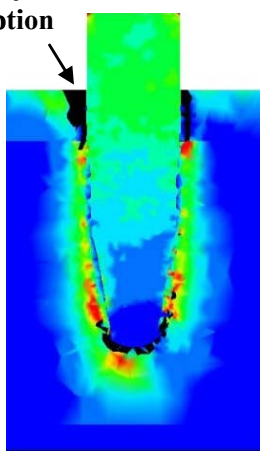

(g) 0.84

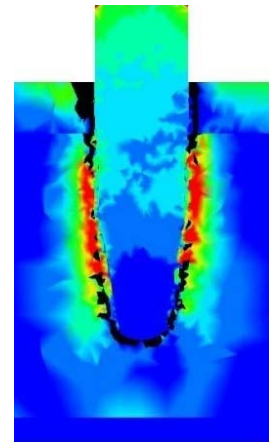

(h) 0.92

Fig. 5 (a) (d) Bucco-lingual and (e) (h) distal-medial cross sectional illustration of the bone resorption patterns under the oblique loading condition simulated according to the equal SED criterion, at a normalized simulation time of (a) and (e) 0.56 ; (b) and (f) 0.70 ; (c) and (g) 0.84 ; (d) and (h) 0.92 . The bone patterns are superimposed on the SED contours.

Figure 5 shows the bucco-lingual and medial-distal cross sections of bone resorption patterns overlapped on the contours of SED at different stages of oblique loading. Clearly, dental bone is resorbed non-symmetrically around the implant. On the bucco-lingual cross section, early bone resorption starts at the upper edge of the cortical bone but at the lingual side (right, Fig. 5a) of the implant, where the SED is most concentrated. Then, bone resorption proceeds downwards to the cancellous bone, until almost the entire lingual side is resorbed (Fig. $5 \mathrm{~b}$ and $\mathrm{c}$ ). Afterwards, the cortical bone at the buccal side begins to be resorbed (left, Fig. 5d). On the medial-distal cross section, however, bone resorption starts almost simultaneously at both distal and medial sides (Fig. 5e and f), though both sides of bone resorption lags behind when compared with the lingual side on the bucco-lingual cross section. After passing the interface and entering the cancellous bone, resorption of the bone 
occurs mostly near the apex of the implant, and extends in the remaining bone adjacent to the implant.

\subsection{Evolution of mechanical fields, change of bone volume and movement of implant}

Bone resorption is caused by the adaptation of bone to its mechanical environment; and in turn the mechanical environment is regulated by bone remodeling and varies with bone resorption. In the present simulations, the evolution of the maximum equivalent stress and strain in the cortical bone and cancellous bone are quantified in Fig. 6. It can be seen that the maximum stresses in both the bones increase almost linearly with the loading time, then they become gradual and transit to a steady stress state of $\sim 34 \mathrm{MPa}$ in the cortical bone and $\sim 11 \mathrm{MPa}$ in the cancellous bone (Fig. 6a). Similarly, the maximum strains in both the bones rise with the loading time, and then gradually evolve into a steady strain state of $\sim 2500 \mu \varepsilon$ in the cortical bone and $7900 \mu \varepsilon$ in the cancellous bone (Fig. 6b).

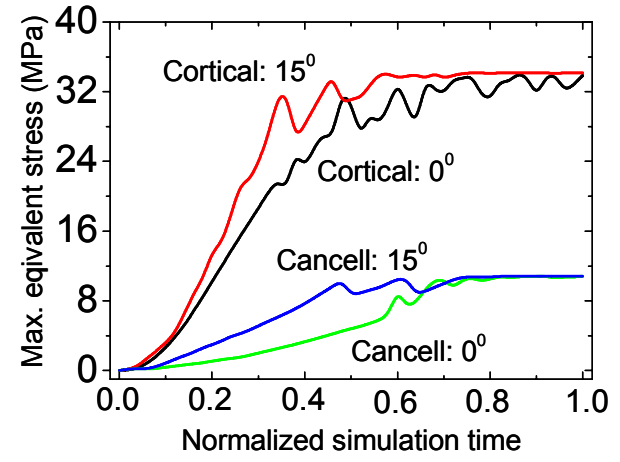

(a)

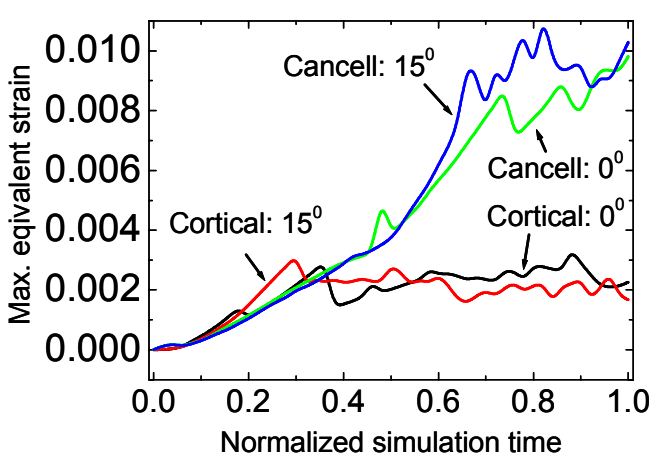

(b)

Fig. 6 The evolution of the maximum equivalent stress (a) and strain (b) in the cortical and cancellous bone under both axial and oblique loading conditions as a function of normalized simulation time.

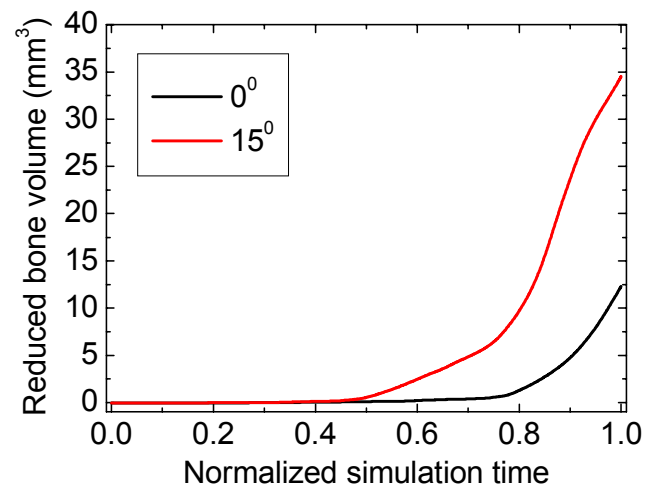

Fig. 7 The variation of resorbed bone volume versus normalized simulation time under axial and oblique loading conditions.

The amount of resorbed bone is increased as the process of bone resorption continues. Fig. 7 shows a quantification of the reduced bone volume as a function of loading time. It can be noted that, at the same time of loading, more bone is resorbed in the case of oblique loading than in the case of axial loading.

Implant movement occurs with bone resorption and causes loosening of the bone. Fig. 8 shows the movement of implant in terms of displacements in three directions versus the loading time. It is noted that, in the case of axial loading, the implant moves downwards vertically only, with the maximum z-displacement of $\sim 25 \mu \mathrm{m}$. However, with increasing the 
time of oblique loading, the $\mathrm{z}$ - and $\mathrm{x}$-displacements of the implant reach as large as $35 \mu \mathrm{m}$ and $50 \mu \mathrm{m}$, respectively, whereas the y-displacement remains negligibly small.

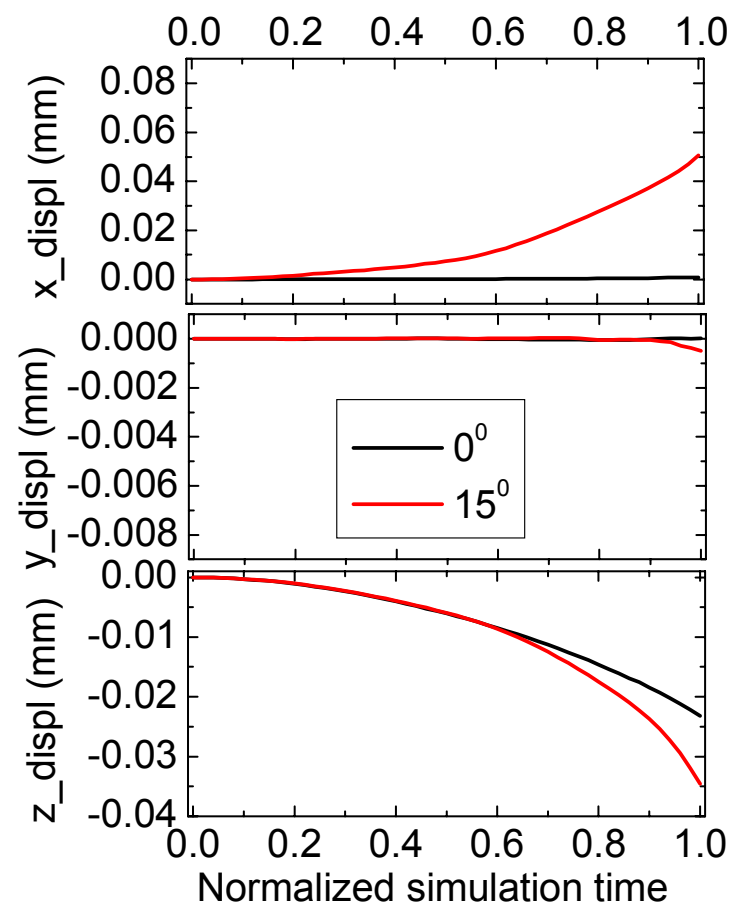

Fig. 8 The variation of implant displacement along $\mathrm{x}, \mathrm{y}$ and $\mathrm{z}$ directions versus normalized simulation time under axial and oblique loading conditions.

\section{Discussions}

Marginal bone resorption has become a major concern in prosthetic implantation, since bone resorption tends to cause the loosening of implant and reduce the success rate of implant treatment. One main reason for the loss of marginal bone around dental implants has been attributed to occlusive overload, which has been evidenced by many clinical and experimental studies ${ }^{(6-8,11)}$. Furthermore, it is reported that the loss of marginal bone is initiated mostly around implant necks and evolves deeper into the bone at later stages ${ }^{(7,8)}$. Finite element analyses of stress and strain fields have indicated that stress concentration occurring exclusively in the cortical bone near the necks of implants is responsible for the initiation of overload-induced bone resorption in this region ${ }^{(12,13)}$. This analysis technique has thus been applied to optimize implant design, with an attempt to improve the biomechanical environment in jawbone/implant systems and reduce bone resorption due to occlusive overload $^{(13-15)}$.

However, few attempts have been made to assess the loosening and movement of implant by simulating the resorption process of dental bone, which is believed to be more informative than mere stress/strain analysis. Although a number of works have been performed, attempting to quantify the process of bone remodeling and bone resorption in long bones such as proximal femur, the emphasis of these works was put on the loss of bone due to stress shielding ${ }^{(10,21)}$. The present simulations, on the other hand, took into account occlusive overload as a major cause for bone resorption, and attempted to reproduce bone resorption patterns that are similar to those observed in realistic situations as in references ${ }^{(6-8)}$. To simulate bone resorption, however, the selection of a reasonable criterion is important. In this work, three criteria, i.e. the equal strain, stress and SED criterion were tested and 
compared. It is found that the equal SED criterion is superior to the other two. This may be interpretable, considering the properties of bones. Both cortical and cancellous bones were assumed to be uniform, each having a different Young's modulus. When the equal strain criterion is used, the cancellous bone, which tends to experience a high strain due to its much lower modulus, easily resorbs first; conversely when the equal stress criterion is used, the cortical bone tends to resorb, because the cortical bone is easily subjected to a higher stress due to its higher modulus. When the cortical bone is fully resorbed based on the equal stress criterion, the stress experienced by the cancellous bone increases, but difficult to reach the threshold value necessary for resorption. On the contrary, the SED criterion, as widely applied to predict damage to materials, is an easily interpretable physical scalar related to both stress and strain, and is therefore capable of reproducing better the pattern and process of bone resorption ${ }^{(21)}$. Here, the SED is assumed to be a mechanical stimulus which bone cells detect; however, the fundamental mechanisms are still unclear, regarding how the bone cells sense and are adapted to the stimulus. And actually, the existence of strain energy sensors has neither been documented in experiments ${ }^{(10,21)}$. Further investigations in this regard are thus much required.

It should be noted, however, that in the present simulation model the geometries of both the cortical and cancellous bones were largely simplified, and the material properties of the bones were assumed to be uniform, isotropic and linear elastic, which might not be as realistic as in actual bone. Further work is now under progress, in which more actual features of jawbone are taken into account to predict jawbone resorption, hopefully more realistic to clinical situations. In addition, various biological factors, which might also contribute to bone resoption and affect the final morphology of the resorbed bone, have not yet been considered in the present research. Moreover, bone resorption due to underload (i.e. disuse of the bone) may also have an influence on the general resorption process. Nevertheless, in spite of these simplifications, the present simulations do provide a qualitative, reasonable understanding of bone resorption phenomena caused by occlusive overload. This enables us to predict bone morphology, stress strain state, as well as the movement of implant at various levels of bone resorption, and thus may supply useful information on optimized design of implant geometry.

\section{Conclusions}

The equal strain energy density criterion has been applied to simulate bone resorption process in a jawbone/implant system and has been demonstrated to be more capable of reproducing jawbone resorption patterns that had been observed in actual clinical situations, when compared to the equal stress or equal strain criterion. It was shown that, under an occlusive loading, bone resorption was initiated at the upper edge of the cortical bone adjacent to the implant. After penetrating the cortical bone along the implant body, the bone resorption passed through the interface between the cortical and cancellous bone and subsequently extended in the cancellous bone. The simulated jawbone morphology after resorption showed a nearly symmetric pattern in the case of axial loading, while it showed an asymmetric prototype in the case of oblique loading. Furthermore, in the case of oblique loading, bone resorption started earlier, and the amount of resorbed bone volume as well as the movement of implant was larger, when compared to the axial loading.

\section{References}

(1) Wennerberg, A., and Jemt, T., Complications in partially edentulous implant patient: a 5-year retrospective follow-up study of 133 patients supplied with unilateral maxillary prosthesis, Clinical Implant Dentistry and Related Research, Vol. 1, (1999), pp.49-56.

(2) Adell, R., Eriksson, B., Lekholm, M., Branemark, P. I., and Jemt, T., A long-term follow-up study of osseointegrated implants in the treatment of totally edentulous jaws, 
The International Journal of Oral \& Maxillofacial Implants, Vol. 5, (1990), pp.347-359.

(3) Ferrigno, N., Laureti, M., Fanali, S., and Grippaudo, G., A long-term follow-up study of nonsubmerged ITI implants in the treatment of totally edentulous jaws. Part I: ten-year life table analysis of a prospective multicenter study with 1286 implants, Clinical Oral Implants Research, Vol. 13, (2002), pp.260-273.

(4) Jemt, T., Lekholm, U., and Adell, R., Osseointegrated implants in the treatment of partially edentulous patients: a preliminary study on 876 consecutively placed fixtures, The International Journal of Oral \& Maxillofacial Implants, Vol. 4, (1989), pp.211-217.

(5) Adell, R., Lekholm, U., Rockler, B., and Brinemark, P.I., A 15-year study of osseointegrated implants in the treatment of the edentulous jaw, International Journal of Oral Surgery, Vol. 10, (1981), pp. 387-416.

(6) Isidor, F., Histological evaluation of peri-implant bone at implants subjected to occlusal overload or plaque accumulation, Clinical Oral Implant Research, Vol. 8, (1997), pp.1-9.

(7) Hoshaw, S.J., Brunski, J.B., and Cochran, G.V.B., Mechanical loading of Branemark implants affects inerfacial bone modeling and remodeling, The International Journal of Oral \& Maxillofacial Implants, Vol. 9, (1994), pp.345-360.

(8) Duyck, J., Ronold, H.J., Van Oosterwyck, H., Naert, I., Vander Sloten, J., and Ellingsen, J.E., The influence of static and dynamic loading on marginal bone reactions around osseointegrated implants: an animal experimental study, Clinical Oral Implants Research, Vol. 12, (2001), pp.207-218.

(9) Frost, H.M., Perspectives: Bone's mechanical usage windows, Bone Mineral, Vol. 19, (1992), pp.257-71.

(10) Huiskes, R., Weinans, H., Grootenbore, H.J., Dalstra, M., Fudala, B., and Slooff, T.J., Adaptive bone-remodeling theory applied to prosthetic-design analysis, Journal of Biomechanics, Vol. 20, (1987), pp.1135-1150.

(11) Quirynen, M., Naert, I., and Van Steenberghe, D., Fixture design and overload influence marginal bone loss and fixture success in the Branemark system, Clinical Oral Implants Research, Vol. 3, (1992), pp.104-111.

(12) Pierrisnard, L., Hure, G., Barquins, M., and Chappard, D., Two dental implants designed for immediate loading: a finite element analysis, The International Journal of Oral \& Maxillofacial Implants, Vol. 17, (2002), pp.353-362.

(13) Qian, L., Todo, M., Matsushita, Y., and Koyano, K., Effects of implant diameter, insertion depth and loading angle on stress/strain fields in implant/jawbone systems, The International Journal of Oral \& Maxillofacial Implants, (2009), in press.

(14) Cynthia, S.P., and John, L.W., Comparative evaluation of implant designs: influence of diameter, length, and taper on strains in the alveolar crest: A three-dimensional finite element analysis, Clinical Oral Implants Research, Vol. 16, (2005), pp.486-94.

(15) Meijer, H.J.A., Huiper, J.H., Starmans, F.J.M., and Bosman, F., Stress distribution around dental implants: Influence of superstructure, length of implants, and height of mandible, Journal of Prosthetic Dentistry, Vol. 68, (1992), pp.96-102.

(16) Beaupre, G.S., Orr, T.E., and Carter., D.R., An approach for time-dependent bone modeling and remodeling-theoretic development, Journal of Orthopaedic Research, Vol. 8, (1990), pp.651-661.

(17) Fyhrie, D.P., and Carter, D.R., Femoral head apparent density distribution predicted from bone stresses, Journal of Biomechanics, Vol. 23, (1990), pp.l-10.

(18) Carter, D.R., Fyhrie, D. P., and Whalen, R.T., Trabecular bone density and loading history: regulation of connective tissue biology by mechanical energy, Journal of Biomechanics, Vol. 20, (1987), pp.785-794.

(19) Prenuergast, P.J., and Taylor, D., Prediction of bone adaptation using damage accumulation, Journal of Biomechanics, Vol. 27, (1994), pp.1067-1076.

(20) Cowin, S.C., and Van Buskirk, W. C., Surface bone remodelling induced by a medullary 
pin, Journal of Biomechanics, Vol. 12, (1979), pp.269-276.

(21) Weinans, H., Huiskes, R., and Grootenboer, H.J., The behavior of adaptive bone-remodeling simulation models, Journal of Biomechanics, Vol. 25, (1992), pp.1425-1441.

(22) Heijer, H.J., Starmans, F.J., Steen, W.H., and Bosman, F., A three-dimensional, finite element analysis of bone around dental implants in an edentulous human mandible, Archives of Oral Biology, Vol. 38, (1993), pp.491-6. 